\title{
Civilisations
}

Revue internationale d'anthropologie et de sciences

humaines

42-2 | 1993

enQuete d'identité

\section{Le voyageur et le nouveau-né : réflexion sur le statut de l'étranger dans les sociétés paysannes roumaines}

Ioana Popescu

\section{CpenEdition}

Journals

Édition électronique

URL : http://journals.openedition.org/civilisations/2355

DOI : 10.4000/civilisations.2355

ISSN : 2032-0442

\section{Éditeur}

Institut de sociologie de l'Université Libre de Bruxelles

\section{Édition imprimée}

Date de publication : 1 décembre 1993

Pagination : 199-204

ISBN : 2-87263-108-9

ISSN : 0009-8140

\section{Référence électronique}

Ioana Popescu, «Le voyageur et le nouveau-né : réflexion sur le statut de l'étranger dans les sociétés paysannes roumaines », Civilisations [En ligne], 42-2 | 1993, mis en ligne le 01 décembre 1996, consulté le 19 avril 2019. URL : http://journals.openedition.org/civilisations/2355 ; DOI : 10.4000/ civilisations.2355

Ce document a été généré automatiquement le 19 avril 2019.

(c) Tous droits réservés 


\title{
Le voyageur et le nouveau-né : réflexion sur le statut de l'étranger dans les sociétés paysannes roumaines
}

\author{
Ioana Popescu
}

1 En Roumanie, le patriotisme, voire l'identité d'appartenance sont fondés sur le sentiment du lieu et sur le culte des ancêtres. C'est pour cela que, dans la mentalité villageoise roumaine,l'étranger n'est pas défini par le critère abstrait de l'appartenance ethnique mais plutôt par celui d'appartenance à la communauté sociale d'un territoire habité. L'étranger est l'inconnu, l'arrivant du dehors. Étrangers sont les membres d'une communauté voisine, plutôt que les covillageois appartenant à une autre ethnie; les artisans spécialisés, marchands, forains, touristes ou... ethnologues, plutôt que les Hongrois, les Allemands ou les Juifs habitant le même territoire.

2 Dans la communauté villageoise roumaine nous et les autres ne forment pas une opposition binaire nette du type bon/mauvais, bénéfique/ maléfique, vrai/faux. Le système de repères connu par la mentalité traditionnelle euphémise ces rapports, en les rendant opérationnels : inconnu/connaissable, contaminé/guérissable, dangereux/apprivoisable.

3 Ce sont justement les contacts avec les autres qui font la différence entre les sociétés «primitives» (qui ne connaissent pas le clivage entre «culture populaire» et «culture savante ») et les sociétés traditionnelles dont l'environnement inclut non seulement la référence aux « ancêtres » mais aussi la culture « savante » des « autres »).

Le plus souvent, l'autre se présente au paysan comme un voyageur. Pour être humain dans une société traditionnelle comme la nôtre, il faut appartenir à un groupe clos. Les connaissances à l'intérieur de ce groupe sont à peu près immuables, alors que les individus évoluent tout au long de leur vie. L'existence individuelle répète les mêmes trajectoires rituelles, selon un savoir acquis directement des ancêtres et transmis d'une génération à l'autre. La liberté individuelle est celle de ne pas devoir s'exprimer soimême, mais d'exprimer ce qui doit être exprimé. 
5 Donc l'individu en tant que tel n'a de sens que par rapport à cette unité minimale qui est le groupe considéré. Dans l'ordre constitué du groupe, le contact avec l'extérieur et avec le voyageur ne doit être mené que sous la forme de négociation. Le propre du voyageur est de marquer la présence d'une absence, d'être le signe visible et accessible d'un monde invisible avec lequel on peut entrer en relation par son intermédiaire. Perçu comme terme de relations différentes, l'autre existe par degrés et comporte des usages différents selon ces relations.

6 L'autre-voyageur, passant qui vient d'un monde différent, est souvent censé être le médiateur des passages rituels entre le monde d'ici et l'au-delà, entre l'homme et les êtres surnaturels, entre une condition existentielle et une autre. Parce qu'il vient du dehors, l'étranger est touché d'impureté, il constitue un danger pour tout le groupe social qui le reçoit. Il doit donc être surveillé attentivement, abordé avec précaution, puis apprivoisé et sédentarisé par des moyens symboliques, pour pouvoir en faire usage. La force magique que lui confère son statut l'amène parfois à devenir l'instrument d'un rite de passage « bénéfique ».

7 Conçue comme succession de métamorphoses, la vie signifie une suite de morts et de résurrections symboliques ; c'est une permanente transgression d'un état périmé vers un autre, actualisé. Au cours de tels passages dangereux, où les mauvais esprits peuvent agir, le voyageur devient alors un agent protecteur.

8 C'est ainsi par exemple que durant la période de gestation, souvent prolongée jusqu'au baptême (les premières semaines après la naissance), on ne doit nommer le petit enfant que par des termes tels que Turc, Petit Turc, Tonneau. Il est donc évident que l'identité conférée par le nom - choisi généralement parmi ceux de la lignée - est gagnée seulement par sa consécration religieuse et que les qualités identitaires de l'enfant doivent se manifester exclusivement sous la protection de Dieu, acquise au Baptême. Les appellations autorisées avant ce moment consistent donc en des noms appartenant à d'autres ethnies ou à d'autres mondes (le monde des objets), des termes catégoriels qui soulignent seulement la qualité d'étranger. En recevant son vrai nom, le nourrisson échange sa position d'étranger sans nom contre le statut, encore incertain, de membre d'une communauté déterminée. Si toutefois il se trouve en péril, l'enfant perd son identité ainsi gagnée et devient Ours, Loup, Terrifiant, noms qui sont censés faire peur aux mauvais esprits qui hantent les frontières de l'au-delà.

9 Mais parfois le voyageur représente un danger pour le nouveau né ; parfois encore il est utilisé comme médiateur, pour capter la bienveillance des Esprits, comme dans les exemples suivants :

- Si un étranger se trouve dans la maison où on allaite un enfant, il ne doit pas sortir sans laisser un signe - un morceau d'étoffe de son vêtement - pour éviter de lui voler le sommeil.

- Pour que l'accouchée ne perde pas son lait, les visiteurs doivent s'asseoir (Sevastos, 1892 : 122).

- L'une des trois Fées du Destin est parfois représentée sous les traits d'une Tsigane. Dans ce cas, c'est elle qui coupe le fil de la vie (informateur village de Boisoara, Banat).

- On aime à raconter aux petits enfants qu'on les a achetés aux Tsiganes en échange d'un tamis de farine de blé ou de maïs (Scurtu, 1924 : 40).

- Le pain qu'on met sur la table des « Ursitoare » (Fées du Destin) doit être pétri par une fille habillée en homme et obligée de se taire pendant tout ce travail (Vitanescu, $1937: 40$ )

- Dans une histoire sur les Fées du Destin, on raconte que celles-ci avaient prédestiné une fille qui venait de naître à épouser l'étranger qui dormait ce jour-là sur le seuil de sa maison. En 
les entendant parler, celui-ci décida de tuer la petite fille et le lendemain il la jeta par dessus les pieux de la palissade. Plus tard il épousa pourtant cette fille qui avait été sauvée et élevée par une étrangère. Le voyageur et l'étranger peuvent donc faire virevolter le destin dans les deux sens grâce à leur positions de médiateurs, à cheval sur deux mondes.

- Trois jours après la naissance, l'accouchée change de chemise. La vieille chemise est donnée en aumône à une vieille femme que l'enfant ne connaîtra jamais (Laugier, 1925 : 81). Le danger est transféré par le groupe social sur l'autre.

- L'enfant doit être allaité pour la première fois par une étrangère assise sur un sac de farine, « sédentarisée » donc par le contact avec la matière consacrée du pain (Revue «Ion Creanga », $1911: 329)$.

- L'enfant qui tête au moins une fois le sein d'une Tsigane ne sera plus jamais victime du mauvais oeil (Revue « Sezatoarea », $1895: 121$ ).

- Si l'enfant va mal, la mère doit porter ses vêtements et son berceau à la frontière du village dans un « espace limite » où le contact avec le même voyageur est possible.

- On dit que si le père de l'enfant fabrique lui-même le berceau, il met son enfant en péril de mort (Scurtu, 1924 : 40).

- Lorsque un nourrisson est très malade ou menacé de mort, la vente rituelle à une femme étrangère ou le choix du parrain dans la rue, sont des rites qui marquent, eux aussi, les qualités de médiateur du non indigène (Simion, 1890 : 102).

- Pour commencer à parler plus tôt, l'enfant reçoit un morceau de pain de la main d'un mendiant, qui lui dit : « Voilà du pain du vieillot pour parler plus tôt » (Simion, 1890 : 336).

- Dans la lignée villageoise, l'orphelin devient toujours « enfant étranger », donc sans identité, comme il est dit dans ces lignes qui l'évoquent:

«Petit raisin gâché par la brume / Qu'il fait mal, mon Dieu, sans mère / Petit raisin gâché par la grêle / Qu'il fait mal, mon Dieu, sans père / Feuille verte d'absinthe / Malheur à l'enfant étranger ".

10 Le don d'une identité individuelle est du ressort du groupe social qui aspire à accorder à ses membres la chance de devenir être de marque ( de seama»).

Être de marque signifie être observé, être vu, être remarqué, se distinguer des autres mais ... tout en gardant la plus forte ressemblance au modèle imposé par la tradition du groupe.

Le processus commence au moment de la naissance, avec les voeux de la sage-femme ( " moasa ») qui soulève l'enfant vers la poutre maîtresse et dit: "Qu'il soit un homme comme il faut et de marque ». Plus significatif est le voeu de la marraine au baptême : «Puisses-tu, filleul, être honoré par tous les hommes, aimé / Comme le sont les saintes icônes »,

Cette notion d'être « de marque » (« de seama ») tend ainsi vers l'exemplarité. Reste à méditer sur l'arrogance de ce dernier voeu, lequel, loin de constituer un blasphème, s'insère, me semble-t-il, dans la conception traditionnelle paysanne du monde ; un monde où, comme le racontent les contes roumains .. même Dieu et saint Pierre voyagent, se fatiguent, maudissent ou gratifient, changeant le cours des choses, comme n'importe quel autre voyageur. L'espace spirituel du paysan les logent tous à la même enseigne, êtres impurs, anges et diables, indigènes et voyageurs ; mais ceux-ci peuvent exister ou non, peuvent gagner ou non une identité, suivant leur façon d'être « de marque ». 


\section{BIBLIOGRAPHIE}

Laugier, Ch. 1925, Contributiuni la etnografia medicala a Olteniei, Craiova, crisuI Românesc.

Scurtu, V., 1924, « Cercetari foIclorice În Ugocea Româna », in : Analele Arhivei de Jolclor , VI, Bucuresti.

Sevastos, E., 1892, Nasterea la români , Iasi.

Simion, FI. M., 1890, Nasterea , Bucuresti.

Vitanescu, P. T., 1937, Monografia comunei Balanesti din Jud. Olt, Craiova.

Rev. Ion Creanga , 1911, $\mathrm{n}^{\circ} 10$.

Rev. Sezatoarea, 1895, $\mathrm{n}^{\circ}$ 5-6.

\section{RÉSUMÉS}

In traditional societies where transition rituals are in use, there always comes a marginal moment, overlapping two worlds, the ancient and the news. At that stage, the two worlds open up cross-cut. The individual subjected to transition rituals then loses his identity, his name, his status.

The newborn baby, coming from the realms of ancestors and entering the land of the living, is welcomed by the community who will make up his identity and go far as give him a «de marque » status, using the "noumenon » of a Stranger. The same agent is used in cases of illness or of death threat, as shown by examples chosen among Rumanian peasant rituals.

\section{INDEX}

Mots-clés : Roumanie, sociétés paysannes, identité, étrangers, rituels

Keywords : Romania, peasant societies, identity, strangers, rituals

\section{AUTEUR}

\section{IOANA POPESCU}

Musée du Paysan Roumain, Bucarest 